\title{
Revista de Relaciones Internacionales, Estrategia y Seguridad
}

\section{El binomio seguridad-desarrollo: algunas aproximaciones interpretativas*}

\author{
John Sebastián Zapata Callejas ${ }^{a}$
}

\begin{abstract}
Resumen: el presente artículo de reflexión busca hacer una reconstrucción teórico-conceptual del binomio seguridad y desarrollo desde un par de marcos interpretativos; los cuales, puntualmente, giran alrededor de dos corrientes explicativas, una primera orientada al accionar internacional y, una segunda, que busca mostrar los ejes articuladores del binomio en la contemporaneidad, el desarrollo humano y la seguridad humana.

En este orden, el texto se va a dividir en cuatro momentos; en el primero, se hace una introducción que sirve como carta de navegación a la problemática; en el segundo, se desarrolla el binomio seguridad-desarrollo desde la lógica del accionar internacional; en el tercero, se interpreta el binomio, en la lógica de sus discursos articuladores modernos: el desarrollo humano y la seguridad humana; finalmente, en el cuarto momento, se harán algunas conclusiones.
\end{abstract}

Palabras clave: Desarrollo; desarrollo humano; seguridad; seguridad humana; relaciones internacionales.

Fecha de recibido: 9/07/2018 Fecha de evaluación: 24/10/2018 Fecha de aprobado: 4/11/2018

Cómo citar: Zapata, J. S. (2019). El binomio seguridad-desarrollo: algunas aproximaciones interpretativas. Revista de Relaciones Internacionales, Estrategia y Seguridad, 14(1), pp. 103-117.

DOI: https://doi.org/10.18359/ries.3556

* Artículo de reflexión asociado a la Maestría en Gerencia para el Desarrollo de la Universidad Externado de Colombia.

a Politólogo de la Universidad de Antioquia. Especialista en Alta Dirección del Estado de la Escuela Superior de Administración Pública (ESAP).

Correo electrónico: zapata0514@hotmail.com ORCID: orcid.org/0000-0002-9468-337X 


\section{The Security-Development Binomial: Some Interpretative Approaches}

Abstract: This reflection paper intends to perform a theoretical-conceptual reconstruction of the security-development binomial from a couple of interpretative frameworks that specifically cover two explanatory trends; a first trend focuses on international action, and a second trend aims at showing the organizational core of the binomial in the contemporary world, human development and human security.

The text consists of four sections. First, there is an introduction that will be the road map to the problem. The second section develops the security-development binomial based on the logic of international action. The binomial is interpreted in the third section following the logic of its modern structuring speeches: Human development and human security. Finally, the fourth section contains some findings.

Keywords: Development; human development; security; human security; international relations.

\section{Binômio segurança-desenvolvimento: algumas abordagens interpretativas}

Resumo: Este artigo de reflexão procura fazer uma reconstrução teórico-conceitual do binômio segurança-desenvolvimento a partir de alguns marcos interpretativos que, pontualmente, giram em torno de duas correntes explicativas, a primeira, que está direcionada à ação internacional e, a segunda, que busca mostrar os eixos articulados do binômio na contemporaneidade, no desenvolvimento humano e na segurança humana.

Nesta ordem, o texto será dividido em quatro momentos. No primeiro, é feita uma introdução, que serve como uma carta de navegação para o problema. No segundo, o binômio segurança-desenvolvimento é desenvolvido a partir da lógica da ação internacional. No terceiro, o binômio é interpretado, na lógica dos seus discursos articuladores modernos: desenvolvimento humano e segurança humana. Finalmente, no quarto momento, algumas conclusões serão feitas.

Palavras-chave: desenvolvimento; desenvolvimento humano; segurança; segurança humana; relações internacionais. 


\section{Introducción}

Para comenzar, se debe decir que al ser el desarrollo un elemento fundamental de las sociedades contemporáneas, el mismo tiene diversos vínculos o nexos con otros componentes sociales, como es el caso de la seguridad. De hecho, desde tiempo atrás se ha venido produciendo en ciertos escenarios de toma de decisiones una convergencia entre las preocupaciones por la seguridad y por el desarrollo, ya que es imposible alcanzar el desarrollo sin una estabilidad, pero a su vez la seguridad no se puede mantener sin desarrollo (Fernández, 2009). ${ }^{1}$

Y es que básicamente desde ciertas cosmovisiones se parte del presupuesto que

la precariedad del desarrollo y la violencia están íntimamente relacionadas, de modo que la inversión en desarrollo favorece la consecución de la paz [...en ultimas la seguridad], y a la vez un ambiente pacífico es favorable para el logro de los objetivos de desarrollo del milenio [... u objetivos de desarrollo sostenible]. (Benavidez, 2010, p. 134)

Es más, algunos actores como el Departamento de Desarrollo Internacional del Reino Unido (DFID) han promovido políticas en las que se expresa que la seguridad y el desarrollo son dos caras de la misma moneda (Woodward \& Taylor, 2005). Así mismo, otros exagentes de agencias internacionales, caso Antonio Mazzitelli, que fuese representante de la Oficina de Enlace y Partenariado de la Oficina de las Naciones Unidas contra la Droga y el Delito (ONUDC) para México, mencionan que

1 Para algunas corrientes la seguridad es la precondición del desarrollo; pero para otras el desarrollo es la precondición del desarrollo. En este orden, por ejemplo, la Fundación para las Relaciones Internacionales y el Diálogo Exterior -Fride- (2008) "la promoción del desarrollo político, económico y social es decisiva para lograr el objetivo final de la seguridad y para evitar que la violencia sea la forma de solucionar los conflictos. De esta manera, desarrollo y seguridad, y sus actores, quedan estrechamente ligados: la seguridad es precondición para el desarrollo y, al mismo tiempo, el desarrollo trabaja para la seguridad" (p. 9); pero, como se ha mencionado, contrario a otros paradigmas es verdaderamente el desarrollo la precondición de la seguridad. Y es quizá esta dualidad una de las problemáticas más arduas en la toma de decisiones contemporáneas, especialmente en los escenarios complejos. "la mejor respuesta y la mejor prevención ante el crimen organizado y su cultura es el desarrollo" (Lucatello, 2013, p. 11).

No en vano, una experta en el tema, Angie Larenas (2012) postula que:

Desde el ámbito del desarrollo se evidencia una preocupación creciente por la guerra y sus efectos. Mientras que el nuevo centro de preocupaciones de la seguridad es el miedo al subdesarrollo como fuente de conflictos, lo que es una manera de justificar el compromiso y la vigilancia continuada: el vínculo entre seguridad y desarrollo. (p. 3)

En este orden de ideas, al observar cierta literatura especializada, se puede concebir que el desarrollo se ha convertido en un ítem fundamental a la hora de hablar de temas como Estados fallidos, Estados débiles, Estados colapsados, gobernabilidad, gobernanza y paz, ${ }^{2}$ los cuales son temarios que tienen relación directa o indirecta con la seguridad; es decir, el desarrollo suele ser un elemento presente cuando se alude a asuntos relacionados con la seguridad y viceversa.

Ahora bien, es interesante analizar que el binomio seguridad y desarrollo se puede comprender estudiando la bibliografía técnica, al igual, que examinando los contextos de ciertos países y determinadas coyunturas nacionales o internacionales, lo que evidencia que existen varias aristas interpretativas sobre dicho binomio. Por ejemplo, una, que es la más tradicional, es la que se desprende del accionar internacional. La misma se ha venido trabajando por décadas debido a la influencia del paradigma hegemónico occidental, el cual promueve el desarrollo y el progreso como fines teleológicos de la civilización, y busca erradicar los contextos tercer mundistas, atrasados y subdesarrollados.

Esta primera arista ha conducido a la implementación de estrategias top-down desde los países

2 Al respecto del tema de la paz, Rafael Grasa (2007) recuerda: “[...] Ahí entra en la agenda la construcción de la paz (peace-building), como resultado de la convergencia entre la nueva agenda de paz y seguridad y la nueva agenda de desarrollo" (p. 10). En otros términos, en la contemporaneidad, cuando se habla de construcción de paz, se involucra transversalmente las agendas de paz y seguridad. 
desarrollados y los organismos multilaterales para ser aplicadas a los países con problemáticas internas, ya que desde el ideario internacional tales Estados representan un peligro para sí mismos, sus vecinos y la sociedad internacional.

Es así como a esta visión clásica de carácter internacional pueden asociársele conceptos como ayuda multilateral, cooperación para el desarrollo, intervencionismo humanitario, ${ }^{3}$ la responsabilidad de proteger, entre otros tantos. Y es que aquí, en definitiva, lo que se pretende es que los países desarrollados brinden ayuda y herramientas a los menos desarrollados para salir de su atraso.

No es de más decir que este derrotero de clase internacionalista sobre el binomio seguridad y desarrollo cuenta con corrientes de naturaleza tanto explicativas como críticas. En otras palabras, una parte de la comunidad científica se ha dedicado a explicar sus dinámicas, pero otra parte se ha orientado a criticarlas, debido a sus implicaciones de índole social, política, económica y cultural. Por ejemplo, Javier Alcalde (2004), postula que:

En efecto, la pretendida vinculación del desarrollo con la paz [y la seguridad], al mismo tiempo que aumentaba a nivel mundial la legitimidad de un proceso de extraordinario interés para el capitalismo, sirvió a los gobiernos de las potencias industriales para justificar en una dimensión moral y universal el instrumento político de la ayuda. Sirvió también a las agencias internacionales para asegurar su propia supervivencia y prosperidad, actuando como vehículos de la ayuda multilateral. (p. 80)

De otro lado, una segunda interpretación, se ubica en la relación explicativa de la seguridad y el desarrollo a la luz de lo que pueden ser los dos discursos articuladores contemporáneos del binomio, la seguridad humana y el desarrollo humano.

Lo anterior, se ampara en el ideario de que, estudiando las agendas y los constructos teóricos de

3 Este concepto es bastante clave en este escrito, por lo mismo cabe destacar que "la intervención humanitaria puede definirse como la intervención militar en un estado, con o sin el consentimiento del mismo, para impedir un genocidio, violaciones a gran escala de los derechos humanos (incluida la hambruna) o violaciones graves del derecho humanitario internacional" (Kaldor, 2010, p. 36). la seguridad y el desarrollo, pareciese que estas dos variables, desde hace un par de décadas, encontraron un par de hilos conductores para solucionar ciertas discrepancias teóricas y prácticas; tal punto se comenzó a dar con el desarrollo humano y prosiguió con la seguridad humana, el hilo conductor más explícito es el de la seguridad humana. En estos dos elementos, por consiguiente, se enmarcan las perspectivas amplias de la teoría desarrollista y la seguritaria, por lo mismo, desde su conceptualización y su hipotética praxis, el binomio seguridad y desarrollo se hace más claro en la dualidad seguridad humana-desarrollo humano.

Al respecto, Larena (2012) postula lo siguiente:

Es en la década de 1990 cuando la influencia entre el desarrollo y la seguridad se vuelve más explícita [...] Se comienza a establecer una relación entre el concepto de seguridad y necesidades humanas, re direccionado también la visión cerrada de las amenazas a la seguridad en términos militares y estatales. Es así como empieza a utilizarse, en la arena policía, el concepto de seguridad humana. (p. 2)

Ahora, si bien se hace necesario analizar y problematizar esta segunda relación, ya que con ella se hace más evidente los nexos contemporáneos del binomio seguridad y desarrollo, es también bastante claro que la misma deja muchas dudas respecto a su postulados teóricos y prácticos, su medición y pragmatismo.

De otro lado, antes de comenzar a dilucidar las dos interpretaciones que pueden extraerse del binomio seguridad-desarrollo, que se desprende de la arista internacional y la de los discursos articuladores, se deben hacer unas precisiones conceptuales que sirvan como marco de referencia para el desarrollo del artículo aquí presente.

Acerca del concepto de desarrollo, vale la pena decir que este concepto ha sido bastante polémico desde sus orígenes en el siglo XX, y su definición depende de la temporalidad, enfoques o teóricos que desarrollen el tema; por ejemplo, los anclados a los paradigmas ortodoxos o convencionales le apuestan a definiciones basadas en el aumento del ingreso per cápita o variables materiales; sin embargo, los heterodoxos o no convencionales se anclan en paradigmas totalmente disimiles. 
Por lo anterior, la profesora Marcel Valcárcel (2006) establece que:

Creemos que siempre habrá tensión entre lo que es y lo que debería ser el desarrollo. El debate entre las distintas versiones sobre el proceso del desarrollo, lo qué es, a quién beneficia y a dónde conduce, por supuesto no está zanjado ni tendría por qué serlo pues el desarrollo, como todo concepto, es necesariamente relativo e histórico. (p. 31)

Es por ello, que se debe traer una definición de desarrollo bastante genérica como la del diccionario de la Real Academia de la lengua española: "por desarrollo debe entenderse la acción y efecto de desarrollar y desarrollarse. Y por desarrollar entiende el aumentar, perfeccionar y mejorar algo $o$ alguien. En tal sentido el concepto engloba una dimensión cuantitativa (aumentar) y una cualitativa (perfeccionar)" (citado por Valcárcel, 2006).

Similar a lo que pasa con el concepto de desarrollo, el termino de seguridad también parte de la concepción que se tenga de la seguridad o el adjetivo que la acompañe, ya sea una visión tradicional centrada en el Estado u otros espacios (seguridad urbana, seguridad ciudadana, seguridad hemisferio, seguridad nacional, entre otros), o una visión centrada en el hombre, como la seguridad humana. Sin embargo, a partir de la misma etimología, donde se sabe que la seguridad deviene del latín securitas/securus, con los cuales se acudía a una situación donde no existían preocupaciones, temores o miedos (Zapata y Sanín, 2013), se puede concluir que la seguridad es esa condición de estar libre de sufrir cualquier afectación, ya sea a nivel a nivel individual, colectivo, territorial o institucional.

Por su parte, la seguridad humana es una tipología de la seguridad no convencional, "se centra en la protección de la persona y las comunidades" (Rojas, 2007, p. 8). Y básicamente lo que busca es ampliar el espectro de las tipologías de las seguridades tradicionales; por ejemplo, el informe del Programa Naciones Unidades para el Desarrollo de 1994 (Informe sobre Desarrollo Humano 1994), establece que la seguridad humana está compuesta por siete seguridades: seguridad economía, seguridad alimenticia, seguridad de salud, seguridad medioambiental seguridad personal, seguridad comunal y seguridad política (Mack, 2005).

En cuanto al desarrollo humano, hay que establecer que este enfoque o tipo de desarrollo no convencional y heterodoxo

tiene como finalidad última a la persona humana y su existencia social; lo importante es la ampliación de oportunidades que en condiciones de igualdad todos tienen; que esas oportunidades están articuladas con las capacidades de las personas. Es decir, lo que la gente es capaz de ser y de hacer. (Arriola, 2007, p. 16)

En últimas, se puede ver al

desarrollo humano como el proceso de ampliación de las opciones de las personas y mejora de las capacidades humanas, es decir, la diversidad de cosas que las personas pueden hacer o ser en la vida, y las libertades para que las personas puedan vivir una vida larga y saludable, tener acceso a la educación, a una vida digna, y a participar en la vida de su comunidad y en las decisiones que los afecten. (Sen, 1998, p. 23)

\section{El binomio seguridad-desarrollo desde la arista internacional}

Para comenzar, se debe establecer que el contexto del binomio seguridad-desarrollo, si bien se puede establecer indirectamente desde los cimientos mismos del discurso del desarrollo en la mitad del siglo XX, el mismo se hizo más tangible y directo en el escenario de la posguerra fría,

en el nuevo horizonte mundial capitalista tras la caída del muro de Berlín se abrió el debate sobre el enfoque de la seguridad, reconstruyendo la agenda de la cooperación al desarrollo para los países del Sur desde el multilateralismo, y reformulando las relaciones de poder entre las redes del Norte en una nueva modulación de la seguridad en el que las exigencias del desarrollo serán la receta para el control de un Sur 'peligrosamente subdesarrollado'. (Zabaleta, 2011, p. 1)

4 Es oportuno resaltar que acá no hay un consenso en las comunidades especializadas sobre tal tema. Por ejemplo, para José Sanahuja (2005), "Seguridad y desarrollo han sido cuestiones que han estado estrechamente entrelazadas desde que aparecen las políticas de ayuda tras la segunda guerra mundial" (p. 2). En otras palabras, para ciertos autores el binomio seguridaddesarrollo han estado estrechamente vinculados desde la segunda 
Y es que se puede notar cierto cambio en la coyuntura de posguerra porque a mediados del siglo $\mathrm{XX}$ y décadas posteriores,

la idea de desarrollo se asociaba exclusivamente al desarrollo económico de los estados. La pobreza, la exclusión social, el hambre y el respeto de los derechos civiles y políticos eran cuestiones internas que los estados debían tratar por si mismos con la única ayuda de la cooperación para el desarrollo de otros países, de las agencias multilaterales y, en casos extremos, de la ayuda humanitaria [...] Las cuestiones de seguridad, por otro lado, tenían una agenda muy limitada centrada en la protección de la integridad territorial, la defensa de la soberanía y la promoción de los intereses nacionales de los estados, siempre en el contexto de la rivalidad bipolar entre el este y el oeste. (Grasa, 2010, p. 72)

En este orden, para Illian (2009), "el concepto de la seguridad ha permeado las agendas políticas de los países donantes" (p. 12), porque se parte del argumento de que se hace necesario la "búsqueda de un orden internacional más o menos estable que permita reducir los conflictos militares, en especial entre países empobrecidos, y los ataques violentos a intereses 'occidentales"' (p. 12).

Adentramos en esta lógica, "aparece el subdesarrollo como la gran amenaza causante del conflicto, la inestabilidad en el mismo sistema mundial" (Zabaleta, 2011, p. 1), porque "ya no son los Estados más fuertes los que son vistos como una amenaza, sino los más débiles. Antes preocupaba la acumulación de poder, ahora la ausencia" (Fernández, 2009, p. 89), como se pensaba en la guerra fría y en otros periodos de la historia.

Ahora bien, desde una perspectiva bastante critica de este andamiaje del desarrollo como la solución global, Ingrid Zabaleta (2011) postula que:

El desarrollo como modelo de progreso societal universalizado por todos los rincones del planeta, resulta

posguerra, para otros autores tal vinculo se hizo realmente claro luego de la caída de Berlín. Otra interpretación la brinda Rafael Grasa (2007): "La Guerra Fría y el mundo bipolar provocaron una separación clara entre las políticas de seguridad y las políticas de desarrollo, articulándose dos arquitecturas político institucionales diferentes, aunque con conexiones entre ellas, para gestionar el desarrollo socioeconómico de los estados y la paz y la seguridad de esos mismos estados y de los sistemas regionales e internacional" (p. 27). el compañero ideal del concepto de seguridad en el nuevo marco de gobernanza mundial, reafirmando el mito colonizador de la cultura y la política de Occidente. (p. 1$)^{5}$

Bajo esta misma lógica José Illan (2009) establece que "no es difícil llegar a la conclusión de que la ayuda al desarrollo se convierte en un instrumento para que los de arriba puedan manejar la inestabilidad que generan los de abajo. (p. 20) ${ }^{6}$

De hecho, otro cumulo de críticas en relación a este binomio se materializan respecto a quién van dirigidas las acciones del escenario global. Un ejemplo claro de esto se puede ver en la ayuda humanitaria para mejorar la seguridad y el desarrollo de los países en contextos complejos, ya que, según ciertos autores, la misma se vuelve selectiva, así lo establece Mark Duffield (2006), “en términos biopolíticos, se necesita separar la vida del desarrollo merecedora de apoyo y de inversión, de una vida humanitaria que puede recibir ayuda, pero también puede ser ignorada hasta su muerte" (p. 13). ${ }^{7}$

5 Al respecto, Mark Duffield (2004) argumenta: "El final de la guerra fría, al remover los cimientos de la arquitectura de la seguridad, ha permitido que el discurso del desarrollo reestablezca sin preocupaciones iniciales por la pobreza y el conflicto. Al mismo tiempo, la política de seguridad se ha reinventado así misma y entre otras cosas, ha llegado a compartir el terreno del desarrollo" (Duffield, p. 66).

6 Al respecto Ingrid Zabalate (2011) dictamina que: "La estrecha relación entre desarrollo y seguridad, profundizara entonces la imposibilidad de que las sociedades que conforman el "peligroso sur subdesarrollo" decidan sobre su propio desarrollo y las alternativas y culturas para el cambio social, manteniendo las relaciones actuales de poder" (p. 1).

7 Apropósito de estas perspectivas críticas, José Illan (2009) recuerda: "En este sentido, el nexo seguridad-desarrollo no es tan claro, por lo menos desde el punto de vista cuantitativo: si la inseguridad es generada en mayor medida por los Estados frágiles, debería destinarse más ayuda para ellos" (p. 17). Por su parte, Larenas (s. f.) menciona: "En últimas, El vínculo no es tacito ni unidimensional, pero tampoco es teórica y políticamente neutro. El desarrollo se securitiza y se convierte en un concepto central para la transformación de aquellas sociedades periféricas institucional y económicamente débiles" (p. 30). Esta anterior problemática, desde cierta interpretación, no es más que la instrumentalización de la ayuda al desarrollo, como de lo demarca Mark Duffield (2004), “La asistencia al desarrollo se politizó durante la guerra fría según las directrices de la estrategia de seguridad dominante, que requerían el establecimiento y el mantenimiento de alianzas políticas prooccidentales entre los países del tercer 
No obstante, más allá de la visión crítica o reafirmativa de la consolidación del binomio en la arista internacional y la influencia en las dinámicas del globo, es innegable que desde hace unos años diversos actores de poder en las relaciones internacionales vienen insistiendo en la importancia de dicho binomio para un mundo más estable, no en vano "una parte de la Ayuda Oficial al Desarrollo (AOD) se destina a financiar actividades ligadas a la seguridad" (Illan, 2009, p. 12).

En este orden, y al identificar la génesis de la relación entre seguridad y desarrollo que ubica al subdesarrollo bajo un status de peligro ${ }^{8}$ para el plano internacional, se comienza a entretejer de manera oportuna y real diversas lógicas o marcos del desarrollo y de seguridad. Así, la promoción del binomio se va efectuando, como se ha insistido, a través de un sistema de cooperación para el desarrollo, el cual tiene como finalidad contrarrestar las múltiples amenazas a la seguridad internacional encontradas en el subdesarrollo (Gómez, 2013). En definitiva, el binomio al ver al subdesarrollo como algo altamente peligroso y dañino utiliza herramientas como la cooperación para contrarrestar tal amenaza.

De hecho, este binomio ha contado con tanta incidencia en temas de cooperación, inter-

mundo" (p. 66). Así mismo, otra versión crítica es que en ocasiones se privilegia él envió de dineros para rescatar instituciones netamente financieras - es decir variables del desarrollo ortodoxo- por encima de la seguridad humana y medio ambiental, algo que se vuelve, entonces incongruente con ciertos discursos internacionales que privilegian supuestamente lo humano.

8 Sobre esto Mark Duffield (2004) menciona: "La incorporación del conflicto en la corriente dominante de la política de ayuda que tuvo lugar a mediados de los noventas ha funcionado como catalizador de la radicalización del desarrollo. En ese proceso, el desarrollo y la seguridad se han ido funcionando. Al calificar el subdesarrollo como peligroso no solo se dice que es necesario un proceso de translocación social correctivo, sino que se provoca una urgencia y la creencia de que este proceso no puede confiarse nunca más a su propia suerte" (p.30). En definitiva, se establece una "lógica que relaciona subdesarrollo y conflicto [... y que] argumenta que es innegable que los conflicto os violentos provocan grandes costos en desarrollo, al mismo tiempo en que la situación de vulnerabilidad de las poblaciones que viven en la pobreza la convierte en caldo de cultivo de procesos violentos. Por lo tanto, el desarrollo puede, al menos indirectamente, contribuir a la prevención del conflicto y, por lo tanto, a la seguridad" (Larenas, s. f., p. 30). vencionismo y ayuda humanitaria, que la ex secretaria de Estado Condoleezza Rice, establecía

es imposible establecer límites claros y concisos entre nuestros intereses en materia de seguridad, nuestros objetivos de desarrollo y nuestros ideales democráticos en el mundo de hoy. Nuestra diplomacia debe integrar y hacer avanzar todos estos objetivos al mismo tiempo. (citado por Illan, 2009, p. 13)

Es así, que:

hacia mediados de los noventa, el Banco Mundial y las agencias bilaterales de desarrollo tomaron conciencia y empezaron a preocuparse por los inmensos costes de las guerras civiles y los conflictos armados para la economía y las perspectivas de desarrollo debido a las destrucciones materiales, los gatos con fines militares y la incapacidad para devolver el servicio de la deuda [... así entonces] La seguridad y el desarrollo aparecían como inextricables, como las dos caras de la misma moneda. (Fernández, 2009, p. 81)

Por ello, se puede dictaminar que, desde múltiples ángulos,

la comunidad internacional, los Estados donantes y buena parte de los especialistas se considera que las políticas amparadas en un vínculo entre ambos conceptos son potencialmente provechosas para encaminarse hacia los objetivos primarios del bienestar humano y el control y la diminución de las amenazas a la seguridad, lo que redundaría en el logro de una paz duradera. (Larenas, s. f., p. 7)

En este orden, en las últimas décadas es constatable el crecimiento o aumento de las redes estratégicas que unen a los actores de la seguridad con los del desarrollo, donde se observa a académicos, altos mandos militares, ONG, compañías de seguridad privada, organizaciones multilaterales, organizaciones bilaterales, OIG, entes supranacionales, entre otros (Duffield, 2004). ${ }^{10}$

9 Por ejemplo, instituciones internacionales, como el Banco Mundial (2011), sugieren algunos recetarios en relación al binomio: "Para alcanzar la seguridad y el desarrollo en el mundo, es fundamental hallar modos eficaces de ayudar a las sociedades a escapar de nuevos brotes o de ciclos repetidos de violencia, pero esto requiere un profundo replanteo, que incluya revisar el modo en que se evalúan y se gestionan los riesgos" (p. 6).

10 Es claro entonces que "la vinculación entre seguridad y desarrollo comienza a plantearse en estos términos a través de 
No en vano, se han dado agendas globales que han involucrado una multiplicidad de actores para consolidar al binomio seguridad-desarrollo, como un tema de relevancia en la toma de acciones vinculantes o en diversos recetarios instruccionales para ciertos países regiones. En estas agendas, se destaca que "para la década de los 90 nuevos postulados influenciaron el surgimiento de un denominado desarrollo alternativo. El Programa de Naciones Unidas para el Desarrollo (PNUD) se perfiló como el pionero en la promoción de la visión humana del desarrollo" (Gómez, 2013, p. 90) o desarrollo humano, además, y casi paralelamente, esta misma organización se vuelve también pionera del concepto de seguridad humana.

Del mismo modo, cabe destacar que Boutros Boutros Gali, ex secretario general de las Naciones Unidas,

contribuye a la consolidación del binomio con su texto Una agenda para el Desarrollo, en el cual define cinco dimensiones del desarrollo, a saber: 1. Paz como fuente necesaria; 2. Economía como energía del proceso; 3. Medio ambiente como base de la sostenibilidad; 4. Justicia como pilar de la sociedad; 5. Democracia como buena gobernanza [...] Haciendo hincapié en la paz como fuente necesaria del proceso de desarrollo, la seguridad, entendida ahora como aquellas medidas destinadas a la reducción de la vulnerabilidad, se convierte en una herramienta fundamental, consolidándose definitivamente la relación dialéctica entre seguridad y desarrollo. (Gómez, 2013, p. 19)

$\mathrm{Y}$ es que la importancia del binomio se consolidó en la arena internacional, ya que al mismo tiempo que se daban inmensos costes para la economía y para otras esferas sociales debido a la no consolidación del binomio en ciertos territorios, se generaron y establecieron una serie de alarmas mundiales cada vez mayores, por parte de las múltiples agencias y organizaciones internacionales. Por lo mismo, nació la pregunta: ¿Qué sentido tenía el esfuerzo dedicado durante décadas a la

organismos internacional, de Estados y desde las posturas de especialistas, sobre todo, en temas de conflicto o postconflicto" (Duffield en Larenas, s. f., p. 29). ayuda al desarrollo si éste era destruido por las guerras civiles y la deuda de los países en conflicto no podía restituirse? En ultimas, se llegó a la conclusión en todos estos organismos que la seguridad y el desarrollo eran inextricables, dos caras de una misma moneda (Woodward y Taylor, 2005).

Por ello, es que

los países donantes y los bancos para el desarrollo respondieron asumiendo la necesidad de crear una sensibilidad ante el conflicto y establecer políticas de ayuda posconflicto. Se investigaron las causas económicas de las guerras civiles, se desarrollaron herramientas de ayuda para todos los programas y operaciones nacionales, se propusieron sanciones económicas para recortar los fondos a los rebeldes, se impulsó la reforma del sector de la seguridad, se presionó a las instituciones de Bretton Woods para hacer caso omiso de sus abogados y revisar el gasto militar, y se empezó a trabajar con regularidad con las Fuerzas Armadas en las operaciones posconflicto. (Woodward y Taylor, 2005, p. 7)

en otras palabras, se concretó- por parte de actores hegemónicos- que el binomio seguridad- desarrollo era un instrumento para la "mejora" y la "viabilidad" estatal en los países no desarrollados o en vías de desarrollo. ${ }^{11}$

De hecho, algunos autores han destacado en la literatura especializada la practicidad del binomio en los países en situación de anomia. Un caso es Paloma González del Mino (2010) y su texto El reto de la comunidad internacional le la reconstrucción de Afganistán: seguridad y desarrollo, allí se destacan estrategias puntuales de reconstrucción o national-building que le apuntan al binomio seguridad-desarrollo. Tal autora subraya que en el caso de Afganistán se le apuntó, luego de la guerra civil, a desarrollar tres pilares: desarrollo

11 Cabe mencionar que "Las agencias de desarrollo de los países ricos siguen siendo los organismos líderes en el diseño de estrategias para los Estados frágiles o fallidos. La nueva agenda de seguridad es vista como un problema de desarrollo, en el sentido de que es sensible a las políticas y a la ayuda que las agencias bilaterales y multilaterales de desarrollo prestan a los países "en riesgo" y a aquellos que se están recuperando de una guerra" (Woodward y Taylor, 2005, p. 7). 
económico-social, el de la seguridad y el buen gobierno. Esto no es más que una muestra fidedigna de la aplicación a nivel interno de las directrices internacionales para la ejecución de políticas que articulan el desarrollo y la seguridad.

Finalmente, se debe decir que si bien antes de 1990 fue claro que, según Gómez (2013), quien cita a Reid (2001), el "binomio seguridad-desarrollo dividió al mundo bajo dos lógicas políticas: la del desarrollo que concretó la separación entre el norte y el sur, y la de seguridad, que concretó la separación entre el Este y el Oeste" (p. 13), también hay que insistir que estas dos lógicas se "congenian hoy por hoy para formar los imaginarios geográficos que guían las acciones de cooperación internacional" (p. 13).

Por lo mismo, y con base en los postulados de Simon Reid-Henry, es necesario resaltar que en relación a este binomio de la seguridad-desarrollo es necesario "identificar cómo el binomio contribuye a la re-imaginación de los espacios geográficos, y en consecuencia a la reidentificación de los desarrollados (buenos y pacíficos) y los subdesarrollados (peligrosos), así como sus implicaciones políticas" (Reid, 2001, citardo por Gómez, 2013, p. 13).

Pero más allá de todo esto, si algo parece ser claro, es que el desarrollo no se posibilita sin seguridad, gobernabilidad, ${ }^{12}$ capacidades estatales, $y$ otras variables; y así mismo, sucede con la las seguridad. $Y$ es que aunque algunos quieran negarlo, "en la actualidad existe cierto consenso sobre el potencial del vínculo para la gestión de sociedades en conflicto o en procesos de reconstrucción postbélica" (Larenas, s. f., p. 7). Además, lo que no se debe olvidar en los escenarios internacionales, es que "la violencia y el conflicto son grandes obstáculos para el desarrollo, pero son también la pobreza, la desigualdad y la injusticia los que empujan a las sociedades hacia la inestabilidad y la violencia" (Bandrés, 2013, p. 1).

12 Sobre la dualidad desarrollo gobernabilidad, en espacial en los Estados fallidos, véase: Zapata (2014).

\section{El binomio seguridad-desarrollo desde los discursos articuladores contemporáneos}

Para comenzar, se debe señalar que

la convergencia entre la seguridad y el desarrollo constituye una relación transfronteriza; un 'puente espaciotemporal' que, como se ha ido relatando, se hace más o menos evidente dependiendo de la temporalidad. Y es que tal 'puente se despliega cuando la seguridad y el desarrollo de un Estado, grupo de Estados o la comunidad internacional, se inmiscuye en la seguridad y el desarrollo de otro Estado'. (Larenas, 2012, p.4)

Sin embargo, la relación también se hace más o menos explícita dependiendo de la misma noción conceptual que se tenga acerca de estos dos conceptos. Por lo mismo, es de suma importancia señalar que, por ejemplo, esas redefiniciones de la

seguridad en la posguerra fría han buscado superar estas limitaciones al reconocer múltiples referentes distintos al estado- el individuo y los grupos sociales, entre ellos- que también experimentan amenazas a su seguridad; al incorporar nuevos temas no militares, el medio ambiente, las drogas ilícitas, la migración, la pobreza y el SIDA, por ejemplo. (Tickner, 2004, p. 15)

Se debe reiterar,

no es una seguridad basada en el control de sus habitantes por parte del Estado ni del empleo de la fuerza militar, sino una seguridad humana [...] porque se centra en la seguridad de las personas, en sus vidas cotidianas. (Illan, 2009, p. 12)

En resumidas cuentas,

al poner al individuo y a las comunidades en el centro de sus reflexiones, la seguridad humana tiende a ampliar el alcance temático de la seguridad, simplemente porque los individuos se enfrentan a una gama mayor de problemas que el estado y la sociedad. (Tickner, 2004, p. 16) ${ }^{13}$

13 Ahora, hay que aclarar que todo este andamiaje se forjó debido a un nuevo consenso internacional de posguerra fría, "respecto a la vinculación entre paz, seguridad y desarrollo, que puede ser interpretado como un intento de desecuritización y resecuritización en el que la seguridad humana constituiría un horizonte normativo para el cambio de políticas, tratando de superar tanto el legado de políticas de seguridad estatocéntricas y militarizadas 


\section{Es de precisar que}

para Naciones Unidas, por ejemplo, la afirmación del concepto de seguridad humana en tanto 'intento de securitización' ha dado más relevancia política a la agenda del desarrollo y la lucha contra la pobreza y a los actores que la promueven. (Sanahuja \& Schuneman, 2012, p. 22)

Por esto, se hace innegable la relación seguridad humana y desarrollo y/o desarrollo humano.

De igual manera, cabe destacar que casi al mismo tiempo, concretamente un par de años antesde la reconfiguración ontológica de la seguridad, se realizó la redefinición del desarrollo en clave de la heterodoxia y de las corrientes no convencionales de la teoría desarrollista, en donde también se pone en el centro del concepto a las personas; ${ }^{14}$ saliendo así a la luz el termino de desarrollo humano,

la noción de Desarrollo Humano (DH) busca superar la visión meramente económica del desarrollo introduciendo la categorización de los Estados mediante la medición de la calidad de vida de su población. El DH es definido como el proceso de expansión de las capacidades de las personas, las cuales amplían sus opciones y oportunidades. (PNUD, 1990, citado por Gómez, 2013, p.5 $)^{15}$

Este par de replanteamientos han hecho que el binomio seguridad y desarrollo sea más claro en términos discursivos y teóricos, representando una asociación contemporánea entre seguridad humana y desarrollo humano. ${ }^{16}$

de la Guerra Fría, como el sesgo occidental de la clásica 'paz liberal'” (Sanahuja en Sanahuja y Schuneman, 2012, p. 25).

14 Sobre esto Manuela Mesa (2009) recuerda que la seguridad humana, "surge en los 80 y se afianza en los 90 vinculada al concepto de desarrollo humano" (p. 3). En la misma lógica Marco Vinicio y Nazareth Porras (2010) describen: "No obstante, el concepto de seguridad humana también surge ante la evolución teórica en el campo del desarrollo humano plateado por el Programa de Naciones Unidas para el Desarrollo en 1990” (p. 161).

15 Vale mencionar que "El desarrollo humano nos resulta de especial interés por la incidencia que ha tenido sobre el discurso político, por su utilización para la praxis política y por su vinculación con la noción de seguridad humana, con la que comportare objetivos y estrategias" (Larenas, s. f., p. 12).

16 No obstante, los antecedentes de la convergencia entre la seguridad humana y el desarrollo humano "se pueden rastrear de manera empírica, a partir de las políticas implementadas en materia de bienestar humano y neutralización de las amenazas a
En este orden, si bien la idea de seguridad estaba anclada y en ocasiones dependía de las agendas de desarrollo, sin duda eran una seguridad y un desarrollo diferente a los que se dieron con la simbiosis entre el adjetivo de lo humano y la seguridad y el desarrollo (Illan, 2009). Por ello, se debe insistir que con la mutación conceptual a la seguridad humana y al desarrollo humano se ha hecho más intrínseco y se articula de manera más concreta el binomio seguridad y desarrollo. ${ }^{17}$

En tal plano de reconfiguración o de dicha simbiosis se han generado visiones alternativas o tradicionalistas de estos dos conceptos. ${ }^{18}$ Sobre las primeras, no es extraño que para autores como Stern y Öjendal (2010), esta reconceptualización del vínculo sea mucho más útil para desafiar y afrontar las visiones tradicionales sobre cómo debe funcionar el mundo $y$, con ello, dar mucha

la seguridad; desde el punto de vista del discurso, partiendo de la retórica empleada para interrelacionar ambos conceptos; o desde una perspectiva teórica, analizando la evolución conceptual del desarrollo y la seguridad que ha llevado a vincular ambos conceptos" (Larenas, 2012, p. 13 ).

17 Cabe notar que en términos conceptuales hay múltiples visiones del binomio seguridad y desarrollo; Severino Cartagena (1998), expone que hay un vínculo entre la seguridad nacional y el desarrollo, debido a que la nación como tal, asociada de cierta manera a la integración económica y la capacidad de desarrollo de una sociedad, y que estas en definitiva crean la base del desarrollo económico de la sociedad.

18 Hay que aclarar que los críticos del desarrollo ven al desarrollo humano como una noción engañosa que sigue al servicio de ciertos intereses hegemónicos. Del mismo modo pasa en la seguridad humana, ya que en ella hay dos versiones; a la versión restringida, por ejemplo, también se le acusa de ser pro intervencionista y estar al servicio de ciertos poderes globales. En este orden vale decir que "todo análisis de los aportes y las limitaciones de la seguridad humana tiene que tomar en cuenta esos dos enfoques [...o visiones]. [... el enfoque o visión amplio] se centra en la 'libertad frente a la necesidad', esto es, en la satisfacción del desarrollo humano y de un mínimo bienestar (seguridad alimentaria, sanitaria, medioambiental, etc.), y se corresponde a la formulación inicial de la seguridad humana realizada por el PNUD en su Informe sobre Desarrollo Humano de 1994, sostenida también por la Comisión de la Seguridad Humana, liderada por Japón, y su relevante informe La seguridad humana ahora, publicado en 2003. Por su parte, el enfoque restringido se centra en la 'libertad frente al temor', esto es, en la protección ante la violencia física en contextos de conflicto. Su gestación y expansión ha venido de la mano en gran medida de la Red de Seguridad Humana, promovida por Canadá e integrada por varios estados" (Pérez de Armiñon, 2006, p. 60). 
más voz a las personas más vulnerables y buscar otro camino para la justicia social. Por ello, es que este par de teóricos consideran que los enfoques del desarrollo humano y la seguridad humana son dos intentos por poner sobre la mesa propuestas alternativas en relación a las demandas sociales planetarias. Sin embargo, a pesar de la intención de dichos enfoques, el de la seguridad humana y el desarrollo humano, por ensanchar sus áreas de actuación, es importante subrayar que tal proceso no siempre conlleva una ampliación de la agenda que corresponda a una profundización de sus mismos contenidos (Larenas, 2012); en otras palabras, con la idea de lo humano se pueden aumentar más variables y problemáticas del mundo social a problematizar y analizar, pero no siempre se logra tener efectividad aplicativa en la mejoría social, debido a la misma amplitud en sus temarios desarrollados.

Bajo esta lógica, se puede notar que

En el fondo, la utilización del concepto "humano", tanto en seguridad como desarrollo, sugiere la asunción de una responsabilidad trasnacional que va más allá del principio de soberanía de los Estados, y que está plenamente en consonancia con el espíritu renovado de la ONU en la década de 1990. (Larenas, s. f., p. 15)

Es propicio precisar entonces que la relación seguridad humana y desarrollo humano va de la mano con la relevancia del binomio seguridaddesarrollo en la esfera internacional y la toma de decisiones supranacionales, ya que es en las lógicas globales que se ha promovido y promocionado de manera más exacerbada las teorías del desarrollo humano y la seguridad humana.

Así mismo, se debe problematizar que si bien se ve al binomio seguridad y desarrollo, esencialmente el materializado entre seguridad humana y desarrollo humano, como una condición que se debe alcanzar para un mundo mejor y más funcional desde el pensamiento oficial y la práctica de la política internacional, una limitación fundamental en esta lógica es la pervivencia de las perspectivas tradicionales sobre la seguridad, en clave estatalista, y del desarrollo en clave económico (Larenas, s. f.).

Por ello, se debe decir que hay una incongruencia a nivel teórico-práctico, porque muchas entidades e instituciones destacan en sus postulados la importancia, al igual, que la utilidad, del desarrollo humano y la seguridad humana, pero en la puesta en marcha de políticas y acciones vinculantes optan por otros modelos de desarrollo o enfoques securitarios. Por lo que se podría decir que la relación desarrollo humano y seguridad humana es más orientada a escenarios teóricos y discursivos que a la praxis; de hecho, estos discursos, hoy por hoy, son de los más escuchados en el globo, como se ha mencionado.

De otro lado, hay que decir que estos dos conceptos tienen fronteras y limites difusos en su relación, gracias a las ampliaciones de sus agendas en vía de lo humano. Pese a esto, la misma humanización y ampliación de la seguridad y del desarrollo si bien se ha traducido en un entrecruzamiento de objetivos, también ha facilitado su vinculación, y otras situaciones como la instrumentalización en la proyección de políticas bajo intereses y propósitos determinados (Larenas, s. f.). ${ }^{19}$

Ahora bien, se debe hacer énfasis en que la génesis del concepto de la seguridad humana y el desarrollo humano encuentran su cúspide en el escenario político-académico del Informe de Desarrollo Humano de 1994. Empero, lo que produce básicamente este informe es una visibilización del binomio estableciendo la relación intrínseca entre el desarrollo y la seguridad, a partir de la seguridad humana como fin último de los modelos sociales y el desarrollo humano como elemento posibilitador de tal fin (Gómez, 2013). ${ }^{20}$

19 Al respecto, Angie Larenas, establece que "han emergido nociones como a seguridad humana y el desarrollo humano, que han llegado para instalarse en el discurso político, la praxis política y entre los especialistas" (Larenas, s. f., p. 11), pero se debe insistir que este par de idea son más normativa que reales.

20 Sobre esto: "En 1994 se revoluciona los estudios de paz, seguridad y desarrollo mediante la introducción del concepto de Seguridad Humana ( $\mathrm{SH}$ ) en el análisis de la política internacional hecho por parte del Programa de Naciones Unidas para el Desarrollo (PNUD). Su postulado central giró en torno al reconocimiento de una relación dialéctica entre la seguridad y el desarrollo, sosteniendo que no puede existir desarrollo sin seguridad, ni seguridad sin desarrollo" (Gómez, 2013, p. 2). En este orden, en una conferencia realizada en Lyosen, Noruega, en mayo de 1999 se "insistía en una idea que ya estaba presente en el informe del PNUD de 1994: la idea de que la seguridad humana y el desarrollo humano, aun siendo conceptos distintos, están estrechamente 
En este orden, uno de los consultores más relevantes en la historia de Naciones Unidas, Mahbul Ul Haq (1994)

planteo que la seguridad humana es la seguridad de los individuos, que busca garantizar su bienestar y supervivencia en cualquier lugar frente a amenazas como las drogas, la enfermedad, el terrorismo y la pobreza; la seguridad que se asegura a través del desarrollo y no de las armas. (citado por Díaz, 2005, p. 142)

Es por esto que se debe señalar que en cuanto a la lógica de la seguridad humana como eje articulador del desarrollo y la seguridad, y el constructo del desarrollo humano como elemento bisagra para llegar a la seguridad humana, hay que postular, entonces, que los mismos representan una secuencialidad, pues, el desarrollo humano consiste en la ampliación de las oportunidades de la gente; mientras la seguridad humana tiene que ver con la posibilidad de disfrutarlas de manera estable (Fuentes y Rojas, 2005, citado por Vinicio y Porras, 2010).

Sobre dicha secuencialidad, a pesar de que no hay un consenso, si hay una tendencia a ubicar al desarrollo humano como el paso fundamental hacia la seguridad humana: "El concepto de seguridad últimamente ha tendido a centrarse más en los individuos y no en los estados, dando paso a una seguridad a través del desarrollo humano" (PNUD, 1993, citado por Delgado, 2008, p. 120). Por lo mismo, "para fomentar la seguridad humana es

relacionados" (Rodríguez 2005, p. 241). Más recientemente, a estos conceptos se les relaciona con otro, el de los derechos humanos. El informe del Secretario General de las Naciones Unidas, Un concepto más amplio de la libertad: desarrollo, seguridad y derechos humanos para todos (2005), representa la primera vez que estas aras quedan oficialmente vinculadas en el orden de prioridades del mantenimiento de paz mundial "La humanidad no podrá tener seguridad sin desarrollo, no podrá tener desarrollo sin seguridad y no podrá tener ninguna de las dos cosas si no respetan los derechos humanos" (Fride, 2008, p. 9).

21 En relación a esto "el nuevo concepto de seguridad humana significa un cambio de énfasis: ya no se trata tanto de defender el territorio o el Estado, cuanto de salvaguardar la seguridad de las personas en todas sus dimensiones. Además, el PNUD propone que el instrumento fundamental para ello no pueden ser las armas y los ejércitos, sino el desarrollo humano" (Rodríguez, 2005, p. 241). "No restulta sorprendente que la formulación del PNUD de 1994 sobre seguridad humana se concentrara en promover 'seguridad a través de desarrollo humano'” (Krause, 2005, p. 26). necesario promover el desarrollo humano sostenible mediante la paliación de la pobreza absoluta, la prestación de servicios sociales básicos para todos y la consecución de objetivos de desarrollo centrados en las personas" (Rodríguez, 2005, p. 250).

En este punto, cabe advertir que hay otras visiones sobre la relación desarrollo humano y seguridad humana en donde se parte de que la segunda es garante de la primera:

la seguridad humana se acerca a la eliminación o por lo menos a dar un manejo a las situaciones y condiciones de extrema vulnerabilidad bajo las que viven amplios sectores sociales marginados de los procesos de desarrollo. El logro principal de la seguridad humana es asegurar un desarrollo humano corto mediano y largo plazo. (Delgado, 2008, p. 122) ${ }^{22}$

Pese a lo anterior, se debe insistir en la idea de ver a la seguridad humana como el articulador contemporáneo del desarrollo y la seguridad, y al desarrollo humano como el posibilitador de la primera noción. ${ }^{23}$

22 Otra interpretación teórica es la dada por Karlos Pérez de Armiñon (2006): “Añadiremos que la aparición del concepto de seguridad humana también es deudora de otra línea de evolución teórica, la habida en el campo del desarrollo y que dio lugar al paradigma del 'desarrollo humano'. Este es el formulado por el Programa de Naciones Unidas para el Desarrollo (PNUD) en 1990 como un proceso de ensanchamiento de las capacidades y libertades de las personas, en contraposición al concepto clásico del desarrollo, entendido en términos de mero crecimiento macroeconómico. El PNUD, al formular posteriormente la idea de la seguridad humana, la define como la condición para que las personas puedan ejercer tales opciones de forma libre y segura, con una relativa confianza en que las oportunidades de hoy no desaparezcan mañana. Desde esta perspectiva, una y otro están estrechamente interrelacionados y se necesitan mutuamente. La seguridad humana es un requisito para el disfrute del desarrollo humano, que a su vez es necesario para materializarla" (p. 63). Más allá de todas estas interpretaciones, lo que si es que "El concepto de seguridad humana ayudaba a catalizar un proceso, una reestructuración más amplia de los discursos paralelos de seguridad y desarrollo" (Krause, 2005, p. 21).

23 Claramente, sobre debe insistir en los incógnitas que generan estos discursos articuladores, por ejemplo, Rafael Grasa (2007) problematiza lo siguiente: "Si bien se ha planteado que el concepto de seguridad humana era un intento de complementar el concepto de desarrollo humano introduciendo la preocupación por la estabilidad de ciertos bienes o, por otro lado, una manera de ampliar su alcance inicial (el que le dio el PNUD en 1990), introduciendo la preocupación por la seguridad física de las personas, lo cierto es que ha pesado más el papel y el interés 
En este orden de ideas, se insiste que desde la perspectiva de la seguridad humana, en su visión restringida, no es posible alcanzar un desarrollo humano sostenible sin contar con seguridad humana, porque están relacionadas intrínsecamente, en donde el primero es un conducto para llegar al segundo (Krause, 2005). En otras palabras, no es posible lograr una

'libertad de necesidades' sin haber conseguido una 'libertad de temores'. En algunos procesos tal vez sea necesario reivindicar la doctrina de 'seguridad en primer lugar', pero en todos los casos debemos reconocer que no es posible ir muy lejos en la promoción del desarrollo humano si no se presta atención a las necesidades básicas de seguridad. (p. 27)

En definitiva, al partir de que la seguridad humana es una concepción más amplia que la del desarrollo humano, se puede por consiguiente dilucidar que la primera "trata de desarrollar una visión global tenga en cuenta aspectos relacionados directamente con el subdesarrollo: desigualdad, salud, crimen internacional, crecimiento poblacional y degradación medioambiental, por poner algunos ejemplos" (Larenas, s. f., p. 19); con el objetivo consolidar y velar por una serie de herramientas que apunten a certificar el buen vivir y la seguridad de lo humano para las personas del mundo; entre esas herramientas, se puede decir, que la fundamental y la garante de tal andamiaje es el desarrollo humano.

\section{Conclusiones}

Lo primero que se debe decir es que el binomio seguridad-desarrollo en la contemporaneidad es un vínculo fuerte y real en la toma de decisiones y en la influencia del devenir mundial.

Ahora bien, pesé a que no existe consenso tácito de si el binomio comenzó a establecer relaciones directas y verificables luego de la segunda guerra mundial o posterior de la guerra fría, lo que sí es constatable es que, al culminar este

de 'desmilitarizar' los estudios de seguridad. Hace falta investigar más sobre todo ello, dada la naturaleza ambivalente de la reflexión genuinamente de desarrollo sobre la expresión seguridad humana "(p. 76). último escenario bipolar, las políticas internacionales se anclaron de cierta medida a la dualidad desarrollo-seguridad.

En este orden, también se puede dilucidar que desde hace casi ya tres décadas, instituciones como la ONU, el Banco Mundial y actores y entidades de relevancia de Estados Unidos, Reino Unido y otros países occidentales de gran poderío geopolítico, comenzaron a darle una prioridad bastante importante al binomio en la arena internacional.

Por lo mismo, en medio de este panorama, tras la caída del muro de Berlín, comienzan a salir a la luz diversas dudas como qué papel tiene el desarrollo en un escenario de violencia o conflicto armado, escenarios que claramente dificultan territorios estables y seguros. A su vez, también surgen, entonces, inquietudes sobre qué consecuencias trae consigo la inseguridad en la capacidad, o no, de posibilitar el desarrollo en los países del primer y tercer mundo, en especial en estas últimas naciones, debido a que hay una preocupación bastante grande en el globo sobre las nuevas guerras, los círculos de violencia y la inseguridad en los países subdesarrollados y en vías de desarrollo.

Todas estas preguntas se dan gracias a las alarmas que se generan por la existencia de escenarios de conflictos armados, guerras civiles, violencias ciudadanas y de contextos que pareciese que por sí solos no pudieran salir de sus condiciones de precariedad. Sumado a lo anterior, se consolida en el plano mundial la inquietud de que si se ayuda a Estados en situación de conflicto, qué fin y utilidad hay si no se pueden consolidar eficazmente dinámicas desarrollo, gracias a las mismas consecuencias de la inseguridad y la beligerancia de dichos países que, a fin de cuentas, obstruyen la oportuna ayuda y cooperación al y para el desarrollo.

Por todo esto, se ha ido calcando y consolidándose en el imaginario planetario que las políticas de seguridad y desarrollo son provechosas para un futuro equitativo en términos de desarrollo y seguridad. Tal imaginario ha llevado, en definitiva, a que se materialice y consoliden grandes redes (compuestas por Estados, instituciones, actores, ONG, y demás) que apuntan a la promoción 
y aplicación de la seguridad y desarrollo en la toma de decisiones nacionales y supranacionales.

Bajo todo este entramado, se puede decir que desde el plano internacional el binomio seguridad y desarrollo es impulsado por estrategias que buscan atacar la ausencia y coexistencia del binomio en los países subdesarrollados, con herramientas y estrategias como la cooperación internacional, la intervención humanitaria, entre otras; gracias a que se parte de la cosmovisión global de que el desarrollo es el fin último de la sociedad y la seguridad es una situación social idónea para los territorios. Y es que, como se mencionó, el concepto de seguridad permeo la agenda internacional, convirtiendo al subdesarrollo en un riesgo para todos los habitantes del planeta.

Sin embargo, cabe destacar que el binomio y sus herramientas como la cooperación o la intervención no están exceptos de críticas, ya que se le acusa a dicho binomio de estar al servicio de los poderes hegemónicos y determinados intereses particulares.

Empero, es innegable que este tema de la conexión seguridad-desarrollo es fundamental para la mejoría social de ciertos contextos complejos, por ende, solamente analizando tal binomio se pueden responder a las siguientes dudas: ¿cómo se articula la seguridad con el desarrollo, qué herramientas utilizar para efectuar políticas de alto impacto positivo en países inseguros y subdesarrollados?, ¿cuál horizonte normativo entre seguridad y el desarrollo priorizar en determinadas temporalidades caracterizadas por la anomia?, entre otras preguntas.

Por último, cabe destacar que con el aterrizaje de la década de 1990 llegó una redirección conceptual en las nociones de seguridad y desarrollo, consolidándose en los discursos y accionares de varias instituciones, los paradigmas de la seguridad humana y el desarrollo humano. Por lo mismo, se puede decir que desde hace varios años dicha seguridad y desarrollo multidimensional han materializado o articulado de manera más concreta el binomio seguridad y desarrollo.

Pero cabe destacar que esta articulación también ha traído una serie de dudas e interrogantes, ya que de los postulados teóricos de la seguridad humana y el desarrollo humano se puede extraer que son idearios más orientados a lo discursivo que a lo práctico, porque en ocasiones tal praxis se ha hecho compleja por los mismos alcances y retos conceptuales; es decir, debido a la amplitud que trajo consigo la noción de humano en la seguridad y el desarrollo, también se complejizo su materialización y efectividad en la toma de decisiones de estos nuevos postulados.

Además, hay una incógnita teórica que trae consigo este par de discursos articuladores de la seguridad humana y el desarrollo humano, y es que no se sabe a ciencia cierta los límites y diferenciaciones de los dos términos. Por ejemplo, algunas corrientes apuestan a que el desarrollo humano es el facilitador para la consecución de la seguridad humana, lo que convertiría a esta última en una noción de más envergadura; mientras otros parten de posturas totalmente diferentes. Aunque por términos pragmáticos y a partir de las variables de las dos corrientes normativas, la seguridad humana se puede decir que es más amplia que el desarrollo humano.

\section{Referencias}

Alcalde, J. (2004). Los Estados Fallidos: la influencia del desarrollo. Lima: CEDEP.

Arriola, G. (2007). Desarrollo humano: una introducción conceptual. Guatemala: Programa de Naciones Unidas para el Desarrollo.

Banco Mundial. (2011). Informe sobre el desarrollo mundial 2011: Conflicto, seguridad y desarrollo. Washington D.C: Banco Mundial.

Bandrés, E. (2013). Conflicto, seguridad y desarrollo. A propósito del informe del Banco Mundial 2011. Observatorio PSyD. Recuperado de http://catedrapsyd. unizar.es/observatorio-psyd/opina/conflicto-seguridad-y-desarrollo-a-proposito-del-informe-del-banco-mundial-2011.html

Benavides. F. (2010). La construcción de la paz y la fragilidad estatal: una revisión de la literatura. Pensamiento Jurídico, 29, 123-176.

Cartagena, S. (1998). El binomio Seguridad-Desarrollo hoy. Hacia un Concepto Contemporáneo de Seguridad Nacional. Revista de Administración Pública, 98, 117-131. 
Delgado, M. (2008). Reconceptualizando la seguridad: cambio de dilemas y amenazas. Revista de Relaciones Internacionales, Estrategia y seguridad, 2, 135-148.

Diaz, L. (2005). Reflexiones sobre la evolución del concepto de seguridad. Papel político estudiantil, 2, 35-46

Duffield, M. (2004). Las nuevas guerras en el mundo global: La convergencia entre el desarrollo y la seguridad. Madrid: Los libros de la catarata.

Duffield, M. (2006). Continuar matando: Gobernación mundial, humanitarismo y terror. Revista Académica de Relaciones Internacionales, 3, 1-28.

Fernández, E. (2009). ¿Estados Fallidos o Estados en Crisis? Granada: Editorial Colmenares.

Fride. (2008). Explorando conceptos: Seguridad humana y construcción de paz. Madrid: Fride

Gómez, B. (2013). El binomio seguridad-desarrollo: ¿De qué estamos hablando? Revista Multidisciplinaria de la División de Ciencias Sociales, 9, 1-25.

González del Miño, P. (2010). El reto de la comunidad internacional le la reconstrucción de Afganistán: Seguridad y desarrollo. Revista Española de Relaciones Internacionales, 2, 10-29. Recuperado de https://dialnet.unirioja.es/descarga/articulo/4844046.pdf

Grasa, R. (2007). Vínculos entre seguridad, paz, y desarrollo: evolución de la seguridad humana. Revista $C I D O B$ d'Afers Iinternationals, 76, 9-46.

Grasa, R. (2010). Cincuenta años de evolución de la investigación para la paz. Barcelona: Generalitat de Catalunya.

Illan, J. (2009). Los vínculos entre la agenda de seguridad y la ayuda al desarrollo. Revista de Relaciones Internacionales de la UNAM, 105, 11-35.

Kaldor, M. (2010). El poder y la fuerza la seguridad de la población civil en un mundo global. España: Tusquets Editores S.A.

Krause, K. (2005). Seguridad humana ¿ha alcanzado su momento? Papeles, 90, 19-30.

Larenas, A. (s. f.). La evolución entre la seguridad y el desarrollo. Examen crítico. España: Universidad del País Vasco.

Larenas, A. (2012). La evolución del vínculo entre seguridad y desarrollo: debates actuales. Boletín electrónico de Hegoa, 33. Recuperado de http://publicaciones.hegoa.ehu.es/es/publications/302

Lucatello, S. (2013). Seguridad y desarrollo. Foreing Affairs Latinoamérica, 13, 8-17.

Mack A. (2005). El concepto de seguridad humana. Papeles, 90, 11-18.

Mesa, M. (2009). Paz y seguridad. CEIPAZ, Fundación Cultura de Paz. Recuperado de http://www.ceipaz.org/ images/contenido/16_paz_seguridad_castellano.pdf
Pérez de Armiñon, K. (2006). Seguridad humana: conceptos, experiencias y propuestas. Revista CIDOB d'Afers Iinternationals, 76, 59-77.

Rodríguez, J. (2005). Seguridad humana: dadle una oportunidad al concepto. En: Acción política no-violenta una opción para Colombia. Bogotá: Centro Editorial Universidad el Rosario.

Rojas, M. (2007). Seguridad humanan: aportes a la reformulación concepto de seguridad. Madrid: Instituto Universitario General Gutiérrez Medallo (INED).

Sanahuja, J. (2005). Seguridad, desarrollo y lucha contra la pobreza tras el 11-S: los Objetivos del Milenio y la "securitizacion" de la ayuda. Documentación Social, 136. Recuperado de http:// docplayer.es/13097240-Seguridad-desarrollo-y-lucha-contra-la-pobreza-tras-el-11-s-los-objetivos-del-milenio-y-la-securitizacion-de-la-ayuda.html

Sanahuja, J y Schunemann, J. (2012). El nexo entre seguridad - desarrollo: entre la construcción de paz y la securitizacion de la ayuda. En: Construcción de la paz, seguridad y desarrollo. Visiones, politicas y actores. Madrid: Editorial Complutense, S.A.

Sen, A. (1998). Bienestar, justicia y mercado. Barcelona: Ediciones Paidós Ibérica.

Tickner, A. (2004). Securitización de la crisis colombiana: bases conceptuales y tendencias generales. Colombia Internacional, 60, 12-35.

Valcarcel, M. (2006). Génesis y evolución del concepto y enfoques sobre el desarrollo. Perú: PUCP- CISEPA. Recuperado de https://www.uv.mx/mie/files/2012/10/ SESION-6-Marcel-Valcarcel-Desarrollo-Sesion6.pdf

Vinicio, M. y Porras, N. (2010). El marco de la seguridad humana: concepción amplia y restringida. Revista Latinoamericana de Derechos Humanos, 1, 159-170.

Woodward, S. y Taylor, M. (2005). Estados Frágiles: soberanía, desarrollo y conflicto. Madrid: Centro de investigación para la paz (CIP-FUHEM)

Zabaleta, I. (2011). Seguridad y gobernanza global: Develando las contradicciones del desarrollo. Recuperado de: www.munduberriak.org

Zapata, S. y Sanín, A. (2013). Otras perspectivas sobre el conflicto en la comuna 13 de Medellín (Colombia): enfoques biopoliticos, seguritarios y de resistencia civil. Papel Político Estudiantil, 9(1-2), 39-66.

Zapata, S. (2014). La teoría del estado fallido: entre aproximaciones y disensos. Revista de Relaciones Internacionales, Estrategia y Seguridad, 1, 87-111. 
